\title{
Emerging roles of chromatin in the maintenance of genome organization and function in plants
}

\author{
Zaida Vergara and Crisanto Gutierrez ${ }^{*}$ (D)
}

\begin{abstract}
Chromatin is not a uniform macromolecular entity; it contains different domains characterized by complex signatures of DNA and histone modifications. Such domains are organized both at a linear scale along the genome and spatially within the nucleus. We discuss recent discoveries regarding mechanisms that establish boundaries between chromatin states and nuclear territories. Chromatin organization is crucial for genome replication, transcriptional silencing, and DNA repair and recombination. The replication machinery is relevant for the maintenance of chromatin states, influencing DNA replication origin specification and accessibility. Current studies reinforce the idea of intimate crosstalk between chromatin features and processes involving DNA transactions.
\end{abstract}

\section{Introduction}

The nuclear processes that are involved in DNA transactions include complex mechanisms responsible for DNA replication, repair, and recombination (the so-called 3Rs). However, the substrate for these processes is not the naked DNA molecule, but chromatin, a highly structured and dynamic macromolecular entity formed by the association of genomic DNA with histones and non-histone proteins. As a consequence, intimate connections exist between these three basic processes and chromatin structure and dynamics. The chromatin status is equally relevant for transcription, another DNA-based process. This process is highly related to the linear topography of different chromatin states and to the three-dimensional (3D) organization of the genome, which defines territories such as euchromatic and heterochromatic domains.

\footnotetext{
* Correspondence: cgutierrez@cbm.csic.es Centro de Biología Molecular Severo Ochoa, CSIC-UAM, Nicolas Cabrera 1, Cantoblanco, 28049 Madrid, Spain
}

The nucleosome, which is the structural unit of chromatin, consists of a core of eight histone molecules (two each of $\mathrm{H} 2 \mathrm{~A}, \mathrm{H} 2 \mathrm{~B}, \mathrm{H} 3$, and $\mathrm{H} 4$ ) and $147 \mathrm{bp}$ of DNA wrapped around it. In addition, histone $\mathrm{H} 1$ binds to the linker DNA between nucleosomes and plays a crucial role in chromatin compaction [1]. The exchange of canonical histones with variant forms, for example, replacing canonical H3.1 with variant $\mathrm{H} 3.3$, contributes to a very significant increase in the diversity of nucleosome types present in the genome [2-4]. Another element of profound structural and functional relevance is the variety of post-translational modifications that occur in residues located in the histone tails $[5,6]$. These modifications include acetylations, methylations, phosphorylations, ubiquitylations, sumoylations, carbonylations, and glycosylations [5]. In addition to histone modifications, the DNA can be methylated at $\mathrm{C}$ residues, with relevant effects on gene expression [7].

In recent years, advances have been made in our understanding of the complex crosstalk between chromatin, transcriptional activity, genome replication, and repair, as well as in characterizing heterochromatin boundaries. Here, we discuss this progress, with an emphasis on plants, and refer to the interested reader to comprehensive reviews for further details.

\section{Genome topography}

The original observation of distinct sub-nuclear territories, such as the densely condensed regions in the nucleus (chromocenters) [8], has advanced in recent years with the generation of genome-wide maps of dozens of DNA and histone modifications. Multiple combinations of chromatin marks actually occur, so the combinatorial possibilities at a given genome locus are extraordinary. The use of sophisticated computational approaches has not only confirmed the preferential association of certain chromatin marks on a genome-wide scale, but also made it possible to begin to decode the different patterns of 
DNA and histone modifications across the genome. This work has now been completed in recent years for various eukaryotic model genomes, including those of mammal models [9-12], Drosophila melanogaster [13, 14], Caenorhabditis elegans [15], Arabidopsis thaliana [16, 17], and Zea mays [18].

\section{Linear topography}

In Arabidopsis, initial studies that focused on chromosome 4 clearly distinguished four major chromatin states, each with a characteristic combination of histone modifications [16]. Importantly, these chromatin domains, which were scattered along the genome, represented active and repressed genes in euchromatin, silent heterochromatin, and intergenic regions. A more recent study, using genome-wide epigenetic datasets, data on DNA properties such as the GC content, and information on the relative enrichment in canonical histone H3.1 and variant H3.3, identified nine distinct chromatin states defining the entire Arabidopsis genome [17]. These states include those previously reported [16] plus others covering those typical of proximal promoters, transcription start sites (TSS), distal intergenic regulatory regions, and two types of heterochromatin.

The number of possible chromatin states depends on how many variables are considered in the analysis, and it is expected to increase in the future. However, it is remarkable that the current set of chromatin states represents the five major elements that form the genome (Fig. 1a):

1. Proximal promoters and TSS $/ 5^{\prime}$ UTRs (chromatin states 2 and 1 , respectively) are typically characterized by marks that are associated with open and highly accessible chromatin, such as H3K4me2/3, high histone H3.3 and H2A.Z, and low H3.1, and that include highly accessible DNase I sites [19]. A high abundance of H3K36me3 and

(a)

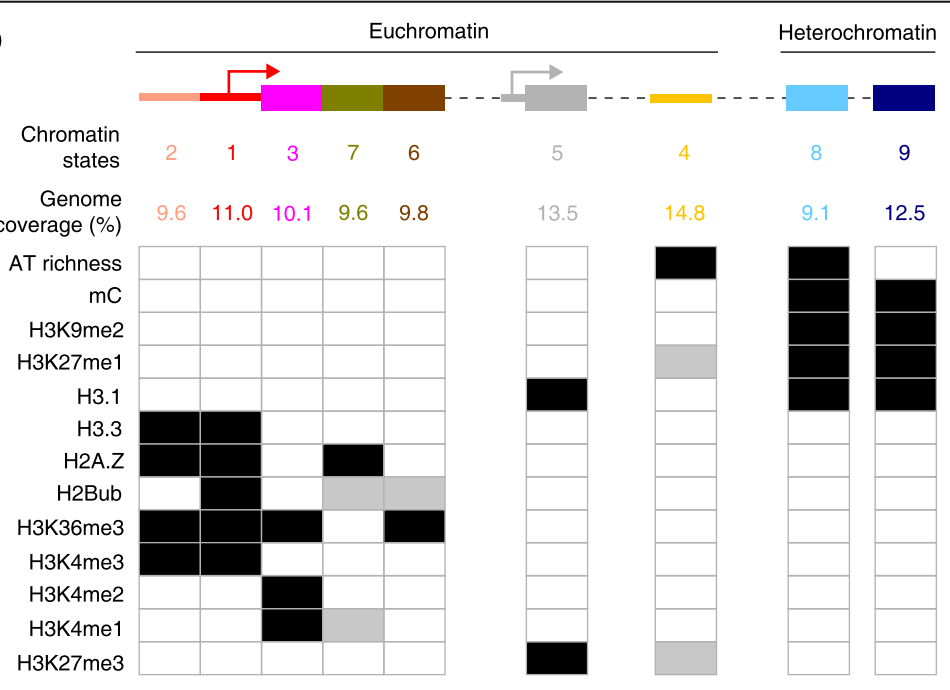

(b)
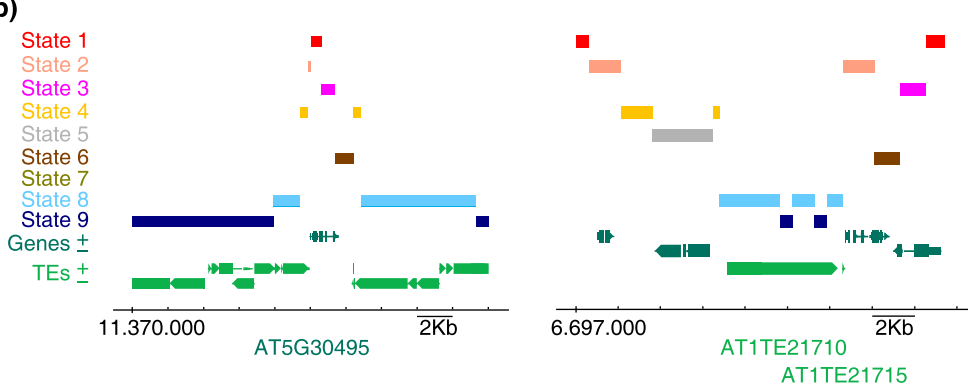

Fig. 1 a The major genomic elements have distinct chromatin states, each characterized by a signature of chromatin marks: proximal promoters (state 2), TSS (state 1), 5' end of genes (state 3), long coding sequences (state 7), 3' end of genes (state 6), polycomb chromatin (state 5), distal regulatory intergenic regions (state 4), AT-rich heterochromatin (state 8), and GC-rich heterochromatin (state 9). The occurrence levels of the main chromatin marks that define each state are summarized as follows: high (black), medium (grey), very low or absent (empty box). b Transitions from euchromatin to heterochromatin states. Left: example of a highly expressed Arabidopsis gene (AT5G30495) that is flanked by repressed transposable elements (TES) in a pericentromeric region of chromosome 5. Right: example of contiguous TEs (AT1TE21710 and AT1TE21715) flanked by expressed genes in one arm of chromosome 1. Note that, in both cases, the transition from repressed heterochromatin (states 8 and 9) to the active euchromatin (state 1) occurs through a defined path of other chromatin states 
H2Bub serves to distinguish chromatin state 1 from state 2 .

2. The genic regions, including the $5^{\prime}$ end, the $3^{\prime}$ end and the long coding sequences, are defined by chromatin states 3,6 , and 7 , respectively. The $5^{\prime}$ end of genes is characterized by relatively high levels of H3K4me1/2 and very low amounts of H3K27me3, whereas at the $3^{\prime}$ end the H3K4me2 modification is almost absent. The coding sequences of long genes may have limited amounts of H3K4me1.

3. The distal regulatory intergenic regions (chromatin state 4) are relatively small due to the compact nature of the Arabidopsis genome, as is also the case in D. melanogaster and C. elegans. Intergenic domains contain moderate levels of H3K27me1 and H3K27me3 and tend to be AT-rich. This domain is also likely to contain many binding sites for transcription factors that act at a distance from the TSS, as recently reported for EIN3 in ethylene signaling [20]. These regions, together with those of chromatin state 2, frequently have properties of bivalent chromatin, containing both H3K4me3 and H3K27me3. Interestingly, these bivalent chromatin regions have been identified in the cells of Arabidopsis plants but only in embryonic animal cells [21]. Full understanding of the biological relevance of this combination of marks awaits a gene-by-gene detailed analysis.

4. Polycomb chromatin has a quite distinct state (state 5), which covers around $13.5 \%$ of the Arabidopsis genome, roughly coinciding with the transcription unit, and is highly enriched in H3K27me3 and histone H3.1.

5. Silent heterochromatin, which is enriched in H3K9me2, H3K27me1, and histone H3.1, among other marks, can be separated into AT-rich (chromatin state 8) and GC-rich (chromatin state 9) heterochromatin. Both forms are largely confined to pericentromeric regions, although there are also scattered patches of heterochromatin in the euchromatic chromosome arms.

A detailed analysis of the neighborhoods in which the nine chromatin states occur revealed the occurrence of prevalent associations. As a consequence, a consensus motif could be deduced that defines the linear topography of the major elements in the Arabidopsis genome (Fig. 1a): promoter and TSS (states 2-1), transcription units (states 3-7-6), Polycomb (state 5), distal regulatory intergenic regions (state 4), and heterochromatin (states 8-9). Remarkably, these associations between domain and chromatin state also correlate nicely with the genomic function of each domain.

\section{Boundaries between chromatin states}

As briefly mentioned above, the chromatin states that define the Arabidopsis genome are non-randomly arranged. It is striking that the propensity of a given state to locate in contact with another is highly dependent on its chromatin signature. Thus, TSS (chromatin state 1) is in contact exclusively with states 2 and 3 (proximal promoters and the $5^{\prime}$ end of genes, respectively). This might be expected, but in other cases, the relationships between chromatin states is surprising. For instance, Polycomb chromatin (state 5) is almost exclusively associated with distal regulatory intergenic regions (state 4), which also contain moderate levels of $\mathrm{H} 3 \mathrm{~K} 27 \mathrm{me} 3$, and with the relatively AT-rich heterochromatin (state 8 ), but not with GC-rich heterochromatin (state 9). Analysis of the linear relationship among all of the chromatin states clearly revealed that chromatin state 4 behaves as a general hub that serves to connect the other chromatin states (equivalent to genomic elements) and that separates the three major chromatin domains: genic regions, Polycomb chromatin, and heterochromatin. In other words, the transition of one of these domains to another does not occur abruptly but rather through a defined and progressive change in chromatin signatures [17]. Interestingly, this also seems to occur in other genomes, such as that of D. melanogaster [14], but the panorama of chromatin states within genomes that share a less compact organization is not currently known.

Arabidopsis has a small and relatively compact genome in which about $36 \%$ of genes are close or immediately adjacent to transposable elements (TEs) [22, 23]. TEs are genomic elements that must be maintained in a silenced and heterochromatic state in most plant tissues, developmental stages, and growth conditions [24, 25]. The constitutive heterochromatic regions are located at the pericentromeric sites, at telomeres, and in the nucleolus organizing regions [26-28]. In addition, there are non-expressed domains within the euchromatic arms that are defined as heterochromatin (that is, enriched in repressive marks). These regions are composed mainly of TEs, inserted within euchromatic regions, and of the polycomb-related genes $[26,29]$.

The physical barriers between heterochromatin and euchromatin form chromatin boundaries, and in Arabidopsis these often occur in the pericentromeric regions. The presence of these boundaries is considered to be a major component of the linear topography of eukaryotic genomes. There are cases in which (i) highly expressed genes are embedded in the highly repressed pericentromeric heterochromatin and flanked by TEs (Fig. 1b, left panel) or (ii) TEs, with the typical repressed chromatin state, are scattered along the euchromatic chromosome arms (Fig. 1b, right panel). As mentioned earlier, the transition from silent heterochromatin to active euchromatin 
(e.g., from state 9 to state 1) does not occur abruptly, but through other chromatin states that cover a relatively small boundary region [17]. Whether a single chromatin mark or a combination of marks defines certain genomic locations as boundaries between euchromatin and heterochromatin is not presently known.

From a mechanistic point of view, different processes have evolved to avoid the spreading of heterochromatin into euchromatin. TE silencing in Arabidopsis results from a combination of the activities of $\mathrm{C}$ methylation pathways that depend on MET1 [30], CMT2/3 [31, 32], and DRM2 as part of the RNA-dependent DNA methylation (RdDM) pathway [33]. (See Box 1 for expansion of abbreviated gene names used in this review.) In addition, the association of heterochromatin domains with the LINC (linker of nucleoskeleton and cytoskeleton) complex in the nuclear periphery is a spatial component that is relevant for heterochromatin silencing, as revealed using loss-of-function mutants [34]. The RdDM pathway, which relies on RNA Pol IV-dependent 24nucleotide short interfering RNAs (siRNAs) [35-37] and RNA Pol V-dependent RNAs [38], is crucial for both preserving the boundaries of heterochromatin domains and keeping TEs silent across generations [18, 39-41]. It has recently been found that the RNA polymerase Pol V is directly involved in defining the edges of TEs. Thus, Pol V transcribes short TEs across their entire length, whereas longer TEs produce Pol V transcripts only at their edges [40]. RNA Pol IV transcripts are also associated with TEs but include both the edges and the TE bodies. More importantly, Pol V, but not Pol IV, transcripts show a high strand preference, being generated from the sense strand at the $5^{\prime}$ end of TEs and from the antisense strand at their 3 ' ends [40]. These data strongly support the idea that Pol $\mathrm{V}$ plays a direct role in defining the heterochromatin boundaries.

In animals, certain histone modifications and related proteins are also involved in defining heterochromatin boundaries; for example, H3K9me $2 / 3$ and HP1 occur at the sites of constitutive heterochromatin and H3K27me3 and the PRC2 complex at facultative heterochromatin [42]. In fission yeast, the HP1 homolog (Swi6) is responsible for preventing the heterochromatic boundaries of the pericentromeric regions, but not of the telomeres, from spreading to the neighboring euchromatic genes [43]. There is evidence that this mechanism also operates in plants. For example, the demethylase IBM1 protects against spreading heterochromatin; in the absence of IBM1, active genes are methylated in the CHG context and accumulate $\mathrm{H} 3 \mathrm{~K} 9 \mathrm{me} 2$ in gene bodies [44] due to the action of KYP and CMT3 [45]. Mutations in the H3K9 methylases, as well as in the LDL2 demethylase, increase H3K4me1 levels in TEs, a prerequisite for TE derepression [44]. Thus, the balance between H3K9me2 and
H3K4me1 appears to be crucial in mediating heterochromatin silencing.

Chromosome 4 of $A$. thaliana (Col-0 ecotype) contains a heterochromatic knob in its short arm, although other accessions, such as Ler, are knobless. The knob was generated by a paracentric inversion, involving two VANDAL5 TEs and two F-box genes, that generated new boundaries between heterochromatin and euchromatin. Studies of DNA methylation, histone methylation, and gene expression have revealed that the epigenetic marks are not modified at the newly generated borders. Instead, the inversion causes linkage disequilibrium with the FRIGIDA gene in the 132 knob-containing accessions identified [46]. Depending on the distance from the insertion of a TE to a gene, the TE can cause heterochromatic signatures to spread to euchromatic genes. This process has been called position-effect variegation in Drosophila [47]. In $A$. thaliana, this process is known to occur in some genes within the heterochromatic knob of chromosome 4. Some of the genes within the knob remain euchromatic and active, whereas others that are close to a VANDAL TE are silent in wild-type plants and active in the $d d m 1$ mutant background [48]. Rice artificial tetraploids show a significant increase in DNA methylation of the $\mathrm{CHG}$ and $\mathrm{CHH}$ contexts that is associated with DNA TEs. More importantly, these DNA methylation changes, linked to alterations in the siRNAs of the RdDM pathway, lead to the repression of genes close to the TEs [49]. The downregulation of these genes, directed by neighbor TE hypermethylation, suggests a possible mechanism for the handling of genedosage effects in polyploid plants.

In plant species whose genomes are larger and more complex than that of $A$. thaliana, the association of TEs with euchromatic domains is more frequent. This is the case, for example, in maize, which has a high TE content and in which $>85 \%$ of genes have a TE within a distance $<1 \mathrm{~kb}$ [50]. In both maize and Arabidopsis, genes are frequently flanked by a relative increase in $\mathrm{mCHH}$, the least common $\mathrm{mC}$ form in genomes, which are known as $\mathrm{mCHH}$ islands [51-53]. Recent studies have revealed that these $\mathrm{mCHH}$ islands play a crucial role in defining the gene/TE boundaries in $>50 \%$ of maize genes [18]. Interestingly, $\mathrm{mCHH}$ islands are mostly located near the inverted repeats of TEs, in particular at the TE edge close to the gene. As this association is more frequent in expressed genes, there is a possibility that different mechanisms for defining gene-TE boundaries may operate depending on the transcriptional status of the affected gene, but it is also clear that the TEs themselves may affect the transcriptional activity of the gene. Studies in maize have demonstrated the role of $\mathrm{mCHH}$ in tagging TE edges near active genes [18]. Thus, mutants that have defects in the MOP1 and MOP3 genes, which encode homologs of the Arabidopsis RDR2 and the large subunit of Pol IV, respectively, 


\section{Box 1. Names of the genes mentioned in this review}

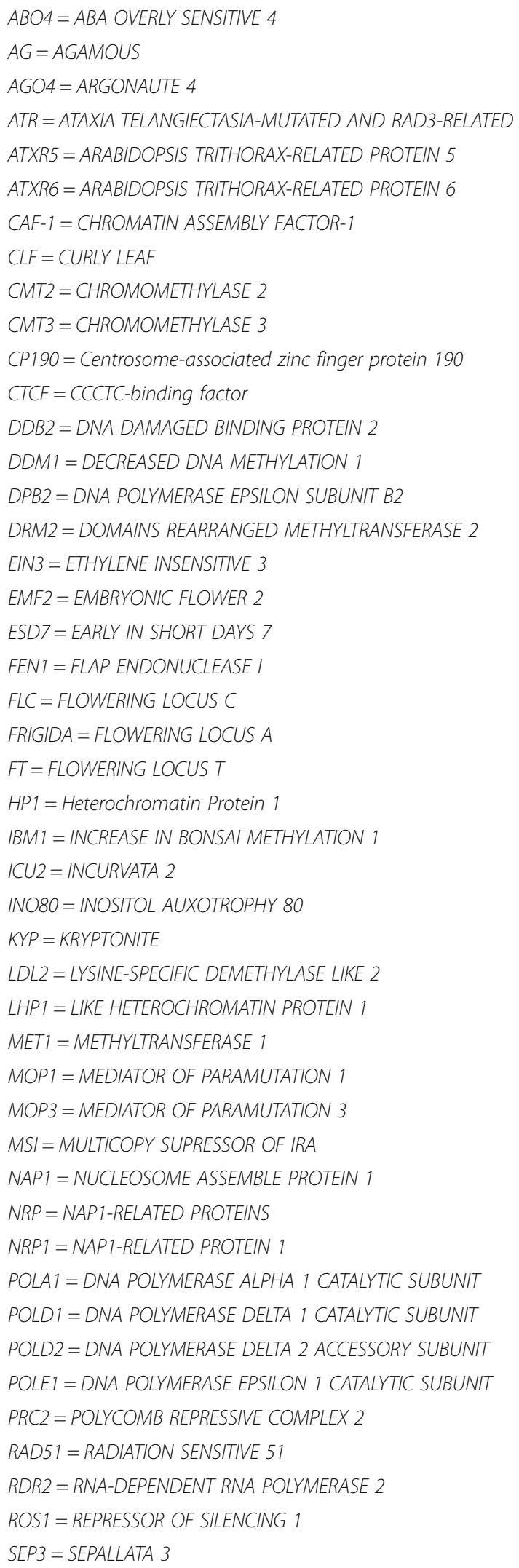

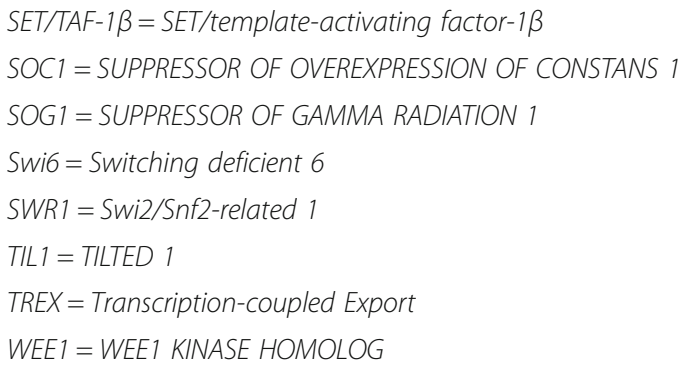

are deficient in $\mathrm{RdDM}$ and in setting appropriate boundaries that prevent an active chromatin state from invading a nearby TE, and vice versa. Furthermore, some maize retrotransposon families show a greater propensity to spread than others, in particular when they are close to genes that are expressed at low levels, pointing to an additional regulatory layer in the control of gene expression [54].

\section{Nuclear territories}

The advances in sophisticated microscopy procedures and analysis, together with recently developed genomic approaches, are contributing to expanding our view of nuclear organization beyond the linear topography of the genome. The so-called 3C (chromosome conformation capture) strategy [55] allows the identification of interactions between one genomic site and many others, and several other genomic procedures have also been developed. These include the $4 \mathrm{C}$ (circular chromosome conformation capture) strategy [56], which determines the interaction of one viewpoint with many genomic locations; the 5C (3C carbon copy) strategy [57], which allows the use of many viewpoints; and the $\mathrm{Hi}-\mathrm{C}$ strategy [58], which is designed to determine the genomic interactions of all loci. The reader is referred to comprehensive reviews for extended discussion of these procedures [59-63]. Here, we highlight only the major discoveries derived from high-throughput genome analysis of chromatin interactions in Arabidopsis [64-68].

A first conclusion of these studies is that the overall 3D interaction network within the Arabidopsis nucleus resembles that of Drosophila and mammalian cells [69] and reveals distinct types of interactions between chromatin states [70]. This is particularly striking for the separation between euchromatin and heterochromatin [64]. In addition, $\mathrm{Hi}-\mathrm{C}$ experiments identified genomic regions that have the general features of active chromatin that establish distal interactions with other similar domains. Short-range interactions also occur between the $5^{\prime}$ and the $3^{\prime}$ end of genes, in particular in highly expressed genes [67]. One largely studied example of such interactions occurs at the FLC locus [71]. By contrast, genomic domains that have the global properties 
of repressed chromatin establish contacts with similar regions and are separated from active domains $[65,66]$. Remarkably, heterochromatic regions-enriched in TEs, H3K9me2 [65], and H3K27me1 [64] - that are interspersed along euchromatic chromosome arms tend to contact each other both in cis and in trans. This leads to the formation of a specific heterochromatin region, called a KNOT [64]. Interestingly, other repressed regions that establish long-range interactions have been reported to contain promoters that are enriched in the H3K27me3 Polycomb mark [67]. This suggests that such interactions might contribute to the coordinated expression of those genes. A recent study, based on the known interaction of the LHP1 protein with H3K27me3 chromatin, has demonstrated that most of the long-range interactions lost in the $\operatorname{lhp} 1$ mutants showed reduced H3K27me3 levels. This, together with expression analysis, revealed the importance of the interaction of LHP1 with H3K27me3 marks in the 3D organization of the Arabidopsis genome and in the coordination of gene expression [68]. The map of spatial interactions in the Arabidopsis genome is increasingly complex, and it has been proposed that plant chromatin adopts various conformations that involve both short-range and long-range interactions (Fig. 2). The various types of looping, including $5^{\prime}-3^{\prime}$ loops and enhancer-promoter loops, as well as the factors affecting chromatin architecture over short- and longranges have been reviewed in detail recently [72].

The formation of genome territories that are well separated by TADs (topologically associating domains), as described for Drosophila $(\sim 100 \mathrm{~kb})$ and mammalian cells (1 Mb) [73-75], does not seem to be a characteristic of the Arabidopsis genome. Owing to the similar sizes of the Arabidopsis and Drosophila genomes, it is perhaps unlikely that the size and compactness of the Arabidopsis genome is the reason for the apparent lack of TADs. Instead, the lack of TADs might be a consequence of the lack in plants of a structural homolog of CTCF in mammals and CP190 in Drosophila [73-75], the proteins that serve as an insulator that defines TAD boundaries [76, 77]. Although typical TADs are missing from Arabidopsis, regions with functional similarities have recently been reported in this plant $[63,66]$. Therefore, it could be very interesting to determine how these TAD-like regions are established and whether they are developmentally regulated or respond to hormonal and environmental cues.

\section{DNA transactions}

Basic cellular processes that are involved in the maintenance and transmission of genetic information actually deal with chromatin, not just naked DNA. Thus, the DNA replication, transcription, repair, and recombination machineries have to act on genome regions containing

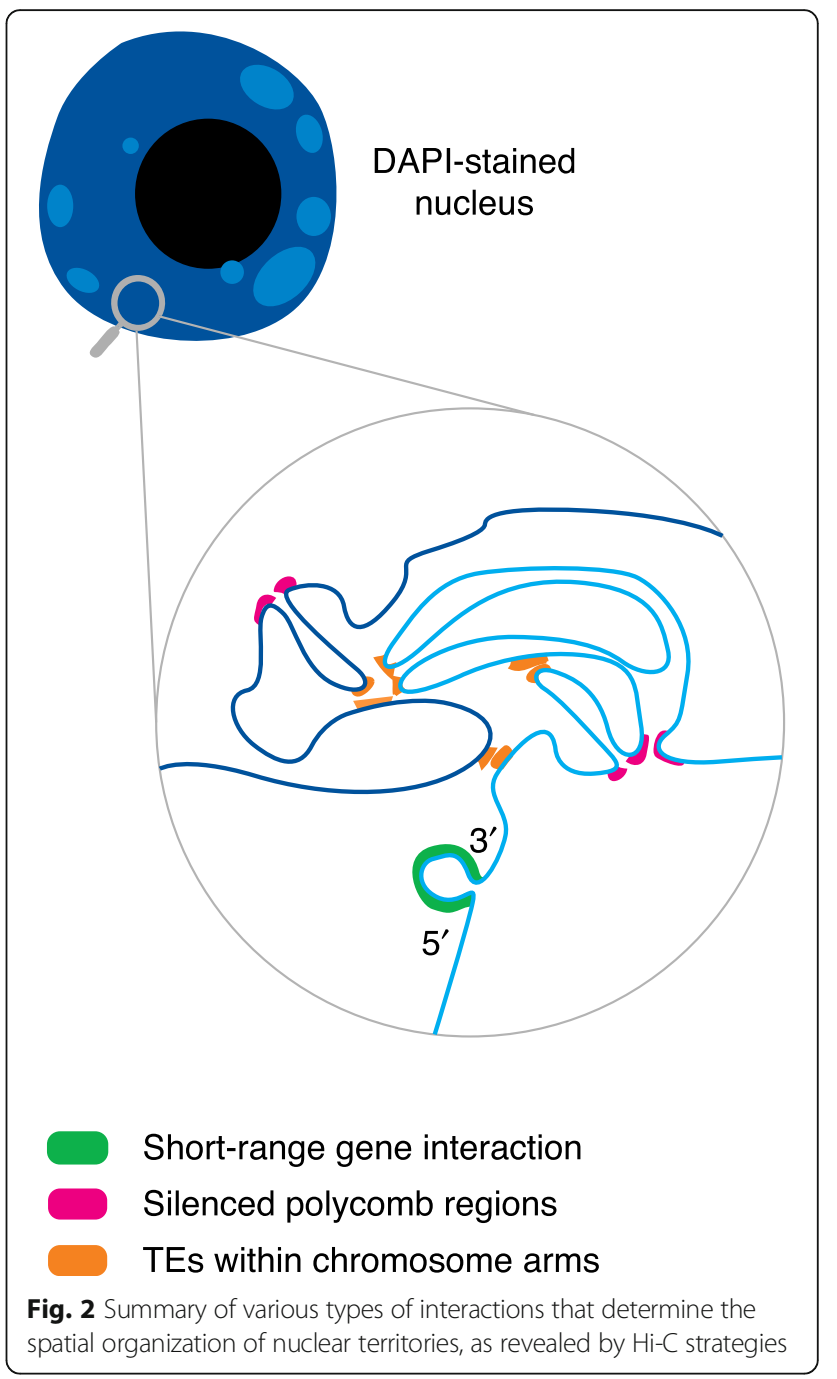

nucleosomes and a plethora of different histone modifications. They need a strict crosstalk with the specific complexes responsible for the disassembly of nucleosomes and their assembly once the process is completed [78]. In addition, the chromatin landscape affects the activity of these macromolecular complexes, which, in turn, also interact with chromatin-modifying complexes. Here, we briefly discuss recent advances on this topic, emphasizing their relevance for genomic and epigenetic maintenance.

\section{Genome replication and chromatin silencing}

The maintenance of epigenetic states is a key aspect of the genome replication process; for example, establishing transcriptional silencing once the replication fork has passed certain genomic regions [79-81]. This silencing is required because histones that are newly deposited by the replicative histone chaperones (CAF1, NAP1, NRP1) do not contain the same set of posttranslational modifications present in parental histones. In some cases, they are actually different isoforms, such 
as canonical H3.1 (as opposed to variant H3.3) because this is the only $\mathrm{H} 3$ deposited by CAF-1 during replication and repair. Remarkably, several components involved in the elongation step during DNA synthesis are directly implicated in transferring epigenetic information to the newly synthesized daughter chromatin strands.

The DNA polymerase $\alpha$, in complex with DNA primase, is responsible for the synthesis of Okazaki fragments in the lagging strand [82], as well as of the first initiation event in the leading strand in each replication origin (ORI). Its large subunit, POLA1, is encoded by the Arabidopsis ICU2 gene [83] and forms a complex, most likely at the replication fork, with CLF and EMF2, components of the PRC2 complex that trimethylates $\mathrm{H} 3$ at residue $\mathrm{K} 27$ [83]. As a consequence, hypomorphic mutations of the ICU2 gene exhibit altered H3K27me3 levels in numerous PRC2 target genes, including the most studied FLC, FT, and $A G$ [84]. POLA1 acts in concert with ROS1, a methylcytosine DNA glycosylase $[85,86]$, to regulate silencing of other loci [87].

DNA polymerase $\delta$ is the holoenzyme complex that extends the lagging strand [82]. POLD1, the large catalytic subunit of this polymerase, is required to maintain correct H3K4me3 levels of certain flowering genes, including FT, SEP3 [88], and probably many others, by mechanisms that are still poorly known. The second largest subunit, POLD2, is also important for the maintenance of transcriptional silencing [89], suggesting that it is the holoenzyme that participates in maintaining a correct balance of H3K4me3 and H3K27me3. This silencing pathway is independent of changes in methylcytosine levels but, interestingly, is dependent on ATR. In fact, pold2-1 mutants are defective in the DNA damage response (DDR) after methyl methanesulfonate (MMS) treatment [89].

DNA polymerase $\varepsilon$ is the third polymerase at the replication fork responsible for the elongation of the leading strand [82]. Its catalytic subunit, POLE1, which is encoded by the POLE1/ABO4/TIL1/ESD7 gene in Arabidopsis [90-92], interacts with CLF, EMF2, LHP1, and MSI [93]. As a consequence, POLE1 participates at the replication fork in the maintenance of the H3K27me3 silencing mark in target genes, including flowering genes such as $F T$ and $S O C 1$, in much the same way as other DNA polymerases. Altered function of DNA Pol $\varepsilon$ in hypomorphic mutants of the large subunit or as achieved by altering the levels of the accessory subunit DPB2 results in hypersensitivity to aphidicolin and hydroxyurea. DPB2 overexpression triggers the expression of DNA repair hallmark genes and produces S-phase lengthening, probably leading to partial genome replication [94]. Genetic analysis has revealed that the DNA Pol $\varepsilon$-dependent pathway is coordinated with ATR, SOG1, and WEE1 to respond to replicative stress [95]. Together, all the data available for various DNA polymerases indicate that the molecular complex responsible for the maintenance of epigenetic states and genome integrity is the whole replisome.

Silencing of TEs that are associated with genome replication occurs through a different molecular pathway. It requires the ATXR5/6 histone methyltransferases that generate $\mathrm{H} 3 \mathrm{~K} 27$ me1 specifically in heterochromatin $[96,97]$. They exhibit a specific activity on the canonical histone H3.1, which is enriched in TEs [98, 99], owing to steric constraints [100]. The atxr5;atxr6 double mutants have defects in controlling DNA replication, as revealed by their abnormal DNA content profiles, which are indicative of DNA over-replication in peri- and nonpericentromeric heterochromatin [101]. This defect occurs preferentially in tissues containing endoreplicating cells, such as cotyledons and old leaves [101, 102]. The double effect of atxr5;atxr6 mutants in transcriptional silencing and DNA replication is an example of replicationtranscription coupling. However, a puzzling observation is that the replication phenotype is suppressed by mutations in the methylcytosine machinery [103], whereas the TE reactivation phenotype is enhanced by the same mutations [102]. This suggests that the transcriptional defects may not be the cause of the replication defects. In fact, decreasing levels of H3K27me1 lead to massive TE transcriptional reactivation resulting from the derepression of TREX activity, which causes an unscheduled excess of transcription to enter into conflict with the replication machinery [102]. One possibility is that an increase in Rloop formation, which has otherwise been linked to the initiation of DNA replication [104], produces replication stress and genome instability.

Biochemical experiments using a whole set of purified yeast replication factors, histones, and chromatin remodeling complexes have directly shown that chromatin organization in the parental strands has profound effects on genome replication efficiency. This occurs at different levels, including ORI selection, the early initiation steps and the replication fork rate $[105,106]$. These experiments demonstrate that the presence of nucleosomes in the parental strands determines various parameters that are crucial for DNA replication. Nevertheless, the existence of different types of nucleosomes, depending on their content in canonical and variant histone forms and on the presence of multiple histone modifications, probably has distinct consequences for the replication process. As discussed earlier, these variables lead to a large combinatorial complexity that has been simplified using computational approaches to identify different chromatin states that are characterized by specific signatures in plants [17] and animals $[12,14,15]$. This information will be instrumental in defining the chromatin landscape of individual ORIs showing different states across the genome. An answer to the question of whether ORIs are associated with one or 
more chromatin signatures awaits the identification of the entire ORI set (the "originome") in a whole organism.

\section{Genome repair and recombination}

The DDR includes, as a first step, the recognition of the DNA lesion. Accessibility to the damaged site is of primary importance and it is significantly affected by the local chromatin landscape. The DDR triggers a cascade of events that lead to the activation of genes required for various forms of DNA repair, depending on the type of DNA damage and the cell cycle stage, among other factors. Both aspects (accessibility and signaling) have been discussed in a comprehensive manner recently [26, 107-109]. Here, we focus on the newest results, with emphasis on how repair and recombination relate to chromatin and vice versa.

The changes in the $\mathrm{H} 3$ and $\mathrm{H} 4$ acetylation patterns that occur soon after X-ray irradiation are a direct indication of DDR at the level of histone modifications, as demonstrated by mass spectrometry [110]. The intimate crosstalk between DDR factors and epigenetic information is relevant during initial DDR events. It was unexpectedly found that plants carrying defects in chromatin remodeling complexes or DNA methylation, such as $d d m 1$ or ros 1 mutants, are also defective in the repair of UV-B DNA damage [111]. Likewise, new roles have recently been found for DDB2, a primary component of the pathway repairing UV-induced DNA damage at the genome level [112]. DDB2 depletion leads to methylation alterations predominantly as the result of a deregulation of the de novo cytosine methylation at centromeric and pericentromeric regions [113]. This is the result of the combined action of (i) DDB2 binding to AGO4, which controls the formation of the 24-nucleotide siRNAs through the RdDM pathway, and (ii) regulation of the expression of the DNA methylcytosine glycosylase ROS1 by DDB2 [113]. Conversely, mutations in DDM1 lead to hypersensitivity to certain DNA-damaging agents [114].

The upregulation of DNA-repair genes is one of the first readouts of DDR activation. ChIP assays have revealed that the increase in gene expression occurs concomitantly with the increase in H3K4me3 levels, particularly around the TSS and gene bodies, without changes in the DNA methylation levels [115]. The gene expression changes in response to DNA damage are not affected, even after knocking out the six genes encoding NAP1 and NRP histone chaperones [116]. This indicates that they participate downstream in the pathway, probably during nucleosome remodeling associated with DNA repair. It has been shown that NAP1 and NRP are required to trigger homologous recombination (HR) before chromatin is remodeled at damaged sites, once $\gamma-\mathrm{H} 2 \mathrm{~A}$.X foci are formed and in an INO80-dependent manner [116]. Recent results show that NRP1 accumulates in chromatin after DNA damage and binds cytochrome c [117] through the NRP1 histone-binding domain [118]. This interaction is important for NRP1 recycling during the disassembly and reassembly of nucleosomes during DNA repair, which parallels the situation with SET/TAF- $1 \beta[119,120]$, the animal functional homolog of Arabidopsis NRP1.

These results are in line with others demonstrating that chromatin remodeling complexes, such as SWR1, which is responsible for depositing H2A.Z, also are relevant for efficient DNA repair, as demonstrated by the reduced levels of repair by HR and the hypersensitivity to DNA-damaging treatments of mutants in which its subunits are defective [121]. It must be emphasized that HR is a very risky process when it occurs in heterochromatin because of the high content of repeated sequences. However, HR predominates over non-homologous end joining (NHEJ) in heterochromatin [26]. One possible way to reduce potential conflicts is to translocate the damaged sites outside the heterochromatin domains, as reported in yeast [122]. However, recent data reveal that Arabidopsis has evolved an alternative pathway whereby pericentromeric heterochromatin undergoes significant remodeling as a consequence of DNA damage produced by over-replication, as, for example, in the atxr5; atxr6 mutant. This allows the formation of unique "over-replicationassociated centers", which have an ordered structure consisting of condensed heterochromatin in the outer layer, the H2A.X variant in another layer, and a core containing $\gamma$ H2A.X and RAD51, possibly among other DNA-repair factors [123]. A recent report strongly suggests evolutionary differences between plants and animals in the H2A proteins associated with DNA repair. Repair of double-strand DNA breaks (DSBs) in the heterochromatin of mammalian cells depends on the phosphorylation of HP1 and KAP1 [124], whereas a different mechanism operates in plants. Thus, in plants, euchromatin DSB repair depends on H2A.X phosphorylation, whereas in heterochromatin repair this role is played by a specific H2A.W7 protein, which is located exclusively in heterochromatin [125] and is phosphorylated by ATM [126].

A correct epigenetic landscape is also necessary for the highly specific recombination events that take place during meiosis. Thus, the level of cytosine methylation strongly affects recombination at crossover hotspots in different ways: (i) RdDM represses crossover formation in euchromatin, increasing nucleosome density and H3K9me2, and (ii) MET1 represses crossover formation in euchromatin and facilitates crossover formation in heterochromatin, as revealed using met1 mutant plants [127].

HR is also a survival mechanism that responds to altered DNA replication fork progression. It requires the correct function of DNA polymerase complexes, as revealed recently for POLD2 and the flap endonuclease FEN1 [89, 128]. The preferential nucleolar accumulation 
of FEN1-GFP poses the question of whether this endonuclease plays a role in genome stability that is related to the organization and copy number of rDNA repeats, an aspect that has not been addressed fully.

\section{Outlook}

Genome organization and function depend heavily on local chromatin properties. The linear topography of chromatin states reveals highly preferred neighborhood associations for the different chromatin states. Why is this necessary and how these preferences are maintained are unanswered questions. In addition, the linear topography facilitates a higher level of complexity by establishing specific domains that have been shown to interact preferentially and to generate a specific organization of nuclear territories in space. Does this simply reflect a structural element of genome organization? Or does it have functional consequences? At least in the case of plants, which have high growth plasticity, it is conceivable that the organization of nuclear domains may change in response to hormonal signals, developmental cues, or environmental challenges. Thus, it is known that the nuclear architecture is modified in response to light during postembryonic development, when heterochromatin reorganization and transcriptional reprogramming are associated with the establishment of photosynthesis [129]. Likewise, epigenetic silencing of TEs is released upon various types of stress, suggesting that the specific chromatin landscapes of silenced TEs, and possibly genes, may regulate their transcriptional response to stress [130].

There are different developmental transitions that are associated with changes in chromatin marks, such as the establishment of a seedling after seed imbibition, the vegetative to reproductive transition, or gametophyte formation. All of these examples rely on changes in H3K27me3 that depend on PRC2 complexes [131]. The gametophytic stage is particularly attractive because of its haploid nature as it is not known whether chromatin states and the organization of nuclear territories depend on ploidy level. Plants contain several dozens of cell types that make up all of their different organs. The individual transcriptomes of all of these cell types have not been obtained yet, but a fair amount of data are becoming available [132-134]. As the transcriptome and the epigenome are intimately linked, the question is whether chromatin states have certain cell-type-specificity. Likewise, a pertinent question is whether changes in the linear topography of the genome have any consequences in the 3D organization of the nucleus. This is a strong possibility, given the preferential association of different genomic regions with similar chromatin signatures.

It is also conceivable that the spatial organization of the nucleus, as well as the local chromatin landscape, impacts the various genome activities that rely on DNA transactions, such as transcription, replication, DNA repair, and recombination [72]. Thus, the epigenome and the transcriptome may affect genome replication dynamics. One of the primary regulatory steps of genome replication is the specification of ORIs. As discussed above, it will be important to identify the originome, which is the collection of all ORIs active in a plant. Efforts to achieve this still face difficulties derived from the limited amounts of short nascent DNA strands purified from replication bubbles and the complexity of the analysis. A future step should aim to identify possible differences in the originomes of specific cell types. This will be a major advance in this field that will open various experimental possibilities to establish links between the originome, the epigenome, and the transcriptome. Systematic and comprehensive studies on these aspects, and surely others, should reveal the mechanisms that relate chromatin and nuclear organization with developmental processes, hormonal responses, and environmental challenges. We look forward to these and many other exciting achievements in this field.

\section{Abbreviations}

3D: Three-dimensional; DDR: DNA damage response; DSB: Double-strand DNA break; HR: Homologous recombination; ORI: DNA replication origin; RdDM: RNA-dependent DNA methylation; siRNA: Short interfering RNA; TAD: Topologically associating domain; TE: Transposable element; TSS: Transcription start site

\section{Acknowledgements}

We thank members of the laboratory for discussions and E. Martinez-Salas for comments. Z.V. was the recipient of a Predoctoral Fellowship FPI from MINECO. This research was supported by grants BFU2012-34821 and BIO2013-50098-EXP from MINECO and BFU2015-68396-R from MINECO/ FEDER, and by an institutional grant from Fundación Ramón Areces to the Centro de Biologia Molecular Severo Ochoa.

Authors' contribution

All authors wrote, read, and approved the final manuscript.

\section{Competing interests}

The authors declare that they have no competing interests.

\section{Publisher's Note}

Springer Nature remains neutral with regard to jurisdictional claims in published maps and institutional affiliations.

Published online: 23 May 2017

\section{References}

1. Happel N, Doenecke D. Histone $\mathrm{H} 1$ and its isoforms: contribution to chromatin structure and function. Gene. 2009;431:1-12.

2. Gurard-Levin ZA, Quivy JP, Almouzni G. Histone chaperones: assisting histone traffic and nucleosome dynamics. Annu Rev Biochem. 2014;83:487-517.

3. Maze I, Noh KM, Soshnev AA, Allis CD. Every amino acid matters: essential contributions of histone variants to mammalian development and disease. Nat Rev Genet. 2014;15:259-71.

4. Otero S, Desvoyes B, Gutierrez C. Histone H3 dynamics in plant cell cycle and development. Cytogenet Genome Res. 2014;143:114-24.

5. Bannister AJ, Kouzarides T. Regulation of chromatin by histone modifications. Cell Res. 2011;21:381-95.

6. Feng S, Jacobsen SE. Epigenetic modifications in plants: an evolutionary perspective. Curr Opin Plant Biol. 2011;14:179-86. 
7. Du J, Johnson LM, Jacobsen SE, Patel DJ. DNA methylation pathways and their crosstalk with histone methylation. Nat Rev Mol Cell Biol. 2015;16:519-32.

8. Heitz E. Das Heterochromatin der Moose. I. Jahrb Wiss Bot. 1928;69:762-818.

9. Hon G, Ren B, Wang W. ChromaSig: a probabilistic approach to finding common chromatin signatures in the human genome. PLoS Comput Biol. 2008; 4 :e1000201.

10. Ernst J, Kellis M. Discovery and characterization of chromatin states for systematic annotation of the human genome. Nat Biotechnol. 2010;28:817-25.

11. McDaniell R, Lee BK, Song L, Liu Z, Boyle AP, Erdos MR, et al. Heritable individual-specific and allele-specific chromatin signatures in humans. Science. 2010;328:235-9.

12. Ernst J, Kheradpour P, Mikkelsen TS, Shoresh N, Ward LD, Epstein CB, et al. Mapping and analysis of chromatin state dynamics in nine human cell types. Nature. 2011;473:43-9.

13. Filion GJ, van Bemmel JG, Braunschweig U, Talhout W, Kind J, Ward LD, et al. Systematic protein location mapping reveals five principal chromatin types in Drosophila cells. Cell. 2010;143:212-24.

14. Kharchenko PV, Alekseyenko AA, Schwartz YB, Minoda A, Riddle NC, Ernst J, et al. Comprehensive analysis of the chromatin landscape in Drosophila melanogaster. Nature. 2011:471:480-5.

15. Liu T, Rechtsteiner A, Egelhofer TA, Vielle A, Latorre I, Cheung MS, et al. Broad chromosomal domains of histone modification patterns in C. elegans. Genome Res. 2011;21:227-36.

16. Roudier F, Ahmed I, Berard C, Sarazin A, Mary-Huard T, Cortijo S, et al. Integrative epigenomic mapping defines four main chromatin states in Arabidopsis. EMBO J. 2011;30:1928-38.

17. Sequeira-Mendes J, Araguez I, Peiro R, Mendez-Giraldez R, Zhang X, Jacobsen SE, Bastolla U, Gutierrez C. The functional topography of the Arabidopsis genome is organized in a reduced number of linear motifs of chromatin states. Plant Cell. 2014;26:2351-66.

18. Li Q, Gent JI, Zynda G, Song J, Makarevitch I, Hirsch CD, et al. RNA-directed DNA methylation enforces boundaries between heterochromatin and euchromatin in the maize genome. Proc Natl Acad Sci U S A. 2015;112:14728-33.

19. Shu H, Wildhaber T, Siretskiy A, Gruissem W, Hennig L. Distinct modes of DNA accessibility in plant chromatin. Nat Commun. 2012;3:1281.

20. Zemlyanskaya EV, Levitsky VG, Oshchepkov DY, Grosse I, Mironova W. The interplay of chromatin landscape and DNA-binding context suggests distinct modes of EIN3 regulation in Arabidopsis thaliana. Front Plant Sci. 2017;7:2044

21. Bernstein BE, Mikkelsen TS, Xie X, Kamal M, Huebert DJ, Cuff J, et al. A bivalent chromatin structure marks key developmental genes in embryonic stem cells. Cell. 2006;125:315-26.

22. Arabidopsis Genome Initiative. Analysis of the genome sequence of the flowering plant Arabidopsis thaliana. Nature. 2000;408:796-815.

23. Ahmed I, Sarazin A, Bowler C, Colot V, Quesneville H. Genome-wide evidence for local DNA methylation spreading from small RNA-targeted sequences in Arabidopsis. Nucleic Acids Res. 2011;39:6919-31.

24. Calarco JP, Borges F, Donoghue MT, Van Ex F, Jullien PE, Lopes T, et al. Reprogramming of DNA methylation in pollen guides epigenetic inheritance via small RNA. Cell. 2012;151:194-205.

25. Negi P, Rai AN, Suprasanna P. Moving through the stressed genome: emerging regulatory roles for transposons in plant stress response. Front Plant Sci. 2016;7:1448.

26. Feng W, Michaels SD. Accessing the inaccessible: the organization, transcription, replication, and repair of heterochromatin in plants. Annu Rev Genet. 2015:49:439-59.

27. Pecinka A, Schubert V, Meister A, Kreth G, Klatte M, Lysak MA, et al. Chromosome territory arrangement and homologous pairing in nuclei of Arabidopsis thaliana are predominantly random except for NOR-bearing chromosomes. Chromosoma. 2004;113:258-69.

28. Schubert V, Berr A, Meister A. Interphase chromatin organisation in Arabidopsis nuclei: constraints versus randomness. Chromosoma. 2012;121:369-87.

29. Fransz $P$, ten Hoopen $R$, Tessadori F. Composition and formation of heterochromatin in Arabidopsis thaliana. Chromosome Res. 2006;14:71-82.

30. Lister R, O'Malley RC, Tonti-Filippini J, Gregory BD, Berry CC, Millar AH, Ecker JR. Highly integrated single-base resolution maps of the epigenome in Arabidopsis. Cell. 2008;133:523-36.

31. Zemach A, Kim MY, Hsieh PH, Coleman-Derr D, Eshed-Williams L, Thao K, et al. The Arabidopsis nucleosome remodeler DDM1 allows DNA methyltransferases to access H1-containing heterochromatin. Cell. 2013;153:193-205.
32. Stroud H, Do T, Du J, Zhong X, Feng S, Johnson L, et al. Non-CG methylation patterns shape the epigenetic landscape in Arabidopsis. Nat Struct Mol Biol. 2014;21:64-72.

33. McCue AD, Panda K, Nuthikattu S, Choudury SG, Thomas EN, Slotkin RK ARGONAUTE 6 bridges transposable element mRNA-derived siRNAs to the establishment of DNA methylation. EMBO J. 2015:34:20-35.

34. Poulet A, Duc C, Voisin M, Desset S, Tutois S, Vanrobays E, et al. The LINC complex contributes to heterochromatin organisation and transcriptional gene silencing in plants. J Cell Sci. 2017;130:590-601.

35. Onodera Y, Haag JR, Ream T, Costa Nunes P, Pontes O, Pikaard CS. Plant nuclear RNA polymerase IV mediates siRNA and DNA methylationdependent heterochromatin formation. Cell. 2005;120:613-22.

36. Herr AJ, Jensen MB, Dalmay T, Baulcombe DC. RNA polymerase IV directs silencing of endogenous DNA. Science. 2005;308:118-20.

37. Pontier D, Yahubyan G, Vega D, Bulski A, Saez-Vasquez J, Hakimi MA, et al, Reinforcement of silencing at transposons and highly repeated sequences requires the concerted action of two distinct RNA polymerases IV in Arabidopsis. Genes Dev. 2005;19:2030-40.

38. Wierzbicki AT, Haag JR, Pikaard CS. Noncoding transcription by RNA polymerase Pol IVb/Pol V mediates transcriptional silencing of overlapping and adjacent genes. Cell. 2008;135:635-48.

39. Fultz D, Choudury SG, Slotkin RK. Silencing of active transposable elements in plants. Curr Opin Plant Biol. 2015;27:67-76.

40. Bohmdorfer G, Sethuraman S, Rowley MJ, Krzyszton M, Rothi MH, Bouzit L, Wierzbicki AT. Long non-coding RNA produced by RNA polymerase V determines boundaries of heterochromatin. Elife. 2016;5:e19092.

41. Fultz D, Slotkin RK. Exogenous transposable elements circumvent identitybased silencing permitting the dissection of expression-dependent silencing. Plant Cell. 2017;29:360-76.

42. Cohen AL, Jia S. Noncoding RNAs and the borders of heterochromatin Wiley Interdiscip Rev RNA. 2014;5:835-47.

43. Stunnenberg R, Kulasegaran-Shylini R, Keller C, Kirschmann MA, Gelman L, Buhler M. H3K9 methylation extends across natural boundaries of heterochromatin in the absence of an HP1 protein. EMBO J. 2015:34:2789-803.

44. Inagaki S, Takahashi M, Hosaka A, Ito T, Toyoda A, Fujiyama A, et al. Genebody chromatin modification dynamics mediate epigenome differentiation in Arabidopsis. EMBO J. 2017;36:970-80.

45. Saze H, Shiraishi A, Miura A, Kakutani T. Control of genic DNA methylation by a jmjC domain-containing protein in Arabidopsis thaliana. Science. 2008; 319:462-5.

46. Fransz P, Linc G, Lee CR, Aflitos SA, Lasky JR, Toomajian C, et al. Molecular, genetic and evolutionary analysis of a paracentric inversion in Arabidopsis thaliana. Plant J. 2016:88:159-78.

47. Elgin SC, Reuter G. Position-effect variegation, heterochromatin formation, and gene silencing in Drosophila. Cold Spring Harb Perspect Biol. 2013;5: a017780.

48. Lippman Z, Gendrel AV, Black M, Vaughn MW, Dedhia N, McCombie WR, et al. Role of transposable elements in heterochromatin and epigenetic control. Nature. 2004:430:471-6.

49. Zhang J, Liu Y, Xia EH, Yao QY, Liu XD, Gao LZ. Autotetraploid rice methylome analysis reveals methylation variation of transposable elements and their effects on gene expression. Proc Natl Acad Sci U S A. 2015;112:E7022-9.

50. Schnable PS, Ware D, Fulton RS, Stein JC, Wei F, Pasternak S, et al. The B73 maize genome: complexity, diversity, and dynamics. Science. 2009:326:1112-5.

51. Gent Jl, Ellis NA, Guo L, Harkess AE, Yao Y, Zhang X, Dawe RK. CHH islands: de novo DNA methylation in near-gene chromatin regulation in maize. Genome Res. 2013:23:628-37.

52. West PT, Li Q, Ji L, Eichten SR, Song J, Vaughn MW, et al. Genomic distribution of H3K9me2 and DNA methylation in a maize genome. PLoS One. 2014;9:e105267.

53. Gent Jl, Madzima TF, Bader R, Kent MR, Zhang X, Stam M, et al. Accessible DNA and relative depletion of $\mathrm{H} 3 \mathrm{~K} 9 \mathrm{me} 2$ at maize loci undergoing RNAdirected DNA methylation. Plant Cell. 2014:26:4903-17.

54. Eichten SR, Ellis NA, Makarevitch I, Yeh CT, Gent JI, Guo L, et al. Spreading of heterochromatin is limited to specific families of maize retrotransposons. PLoS Genet. 2012;8:e1003127.

55. Dekker J, Rippe K, Dekker M, Kleckner N. Capturing chromosome conformation. Science. 2002;295:1306-11.

56. Zhao Z, Tavoosidana G, Sjolinder M, Gondor A, Mariano P, Wang S, et al. Circular chromosome conformation capture (4C) uncovers extensive 
networks of epigenetically regulated intra- and interchromosomal interactions. Nat Genet. 2006;38:1341-7.

57. Dostie J, Richmond TA, Arnaout RA, Selzer RR, Lee WL, Honan TA, et al. Chromosome Conformation Capture Carbon Copy (5C): a massively parallel solution for mapping interactions between genomic elements. Genome Res. 2006:16:1299-309.

58. Lieberman-Aiden E, van Berkum NL, Williams L, Imakaev M, Ragoczy T, Telling A, et al. Comprehensive mapping of long-range interactions reveals folding principles of the human genome. Science. 2009;326:289-93.

59. de Wit $\mathrm{E}$, de Laat W. A decade of $3 C$ technologies: insights into nuclear organization. Genes Dev. 2012;26:11-24.

60. Dekker J, Marti-Renom MA, Mirny LA. Exploring the three-dimensional organization of genomes: interpreting chromatin interaction data. Nat Rev Genet. 2013;14:390-403.

61. Belmont AS. Large-scale chromatin organization: the good, the surprising, and the still perplexing. Curr Opin Cell Biol. 2014;26:69-78.

62. Pombo A, Dillon N. Three-dimensional genome architecture: players and mechanisms. Nat Rev Mol Cell Biol. 2015;16:245-57.

63. Liu C, Weigel D. Chromatin in 3D: progress and prospects for plants. Genome Biol. 2015;16:170.

64. Grob S, Schmid MW, Grossniklaus U. Hi-C analysis in Arabidopsis identifies the KNOT, a structure with similarities to the flamenco locus of Drosophila. Mol Cell. 2014;55:678-93.

65. Feng S, Cokus SJ, Schubert V, Zhai J, Pellegrini M, Jacobsen SE. Genomewide $\mathrm{Hi}-\mathrm{C}$ analyses in wild-type and mutants reveal high-resolution chromatin interactions in Arabidopsis. Mol Cell. 2014;55:694-707.

66. Wang C, Liu C, Roqueiro D, Grimm D, Schwab R, Becker C, et al. Genomewide analysis of local chromatin packing in Arabidopsis thaliana. Genome Res. 2015;25:246-56

67. Liu C, Wang C, Wang G, Becker C, Zaidem M, Weigel D. Genome-wide analysis of chromatin packing in Arabidopsis thaliana at single-gene resolution. Genome Res. 2016:26:1057-68.

68. Veluchamy A, Jegu T, Ariel F, Latrasse D, Mariappan KG, Kim SK, et al. LHP1 regulates $\mathrm{H} 3 \mathrm{~K} 27 \mathrm{me} 3$ spreading and shapes the three-dimensional conformation of the Arabidopsis genome. PLoS One. 2016;11:e0158936.

69. Sequeira-Mendes J, Gutierrez C. Genome architecture: from linear organisation of chromatin to the 3D assembly in the nucleus. Chromosoma. 2016;125:455-69.

70. Wang J, Zhou Y, Li X, Meng X, Fan M, Chen H, et al. Genome-wide analysis of the distinct types of chromatin interactions in Arabidopsis thaliana. Plant Cell Physiol. 2017:58:57-70.

71. Crevillen P, Sonmez C, Wu Z, Dean C. A gene loop containing the floral repressor $F L C$ is disrupted in the early phase of vernalization. EMBO J. 2013;32:140-8.

72. Rodriguez-Granados NY, Ramirez-Prado JS, Veluchamy A, Latrasse D, Raynaud C, Crespi M, et al. Put your 3D glasses on: plant chromatin is on show. J Exp Bot. 2016;67:3205-21.

73. Dixon JR, Selvaraj S, Yue F, Kim A, Li Y, Shen Y, et al. Topological domains in mammalian genomes identified by analysis of chromatin interactions. Nature. 2012:485:376-80

74. Nora EP, Lajoie BR, Schulz EG, Giorgetti L, Okamoto I, Servant N, et al. Spatial partitioning of the regulatory landscape of the $\mathrm{X}$-inactivation centre. Nature. 2012;485:381-5

75. Sexton T, Yaffe E, Kenigsberg E, Bantignies F, Leblanc B, Hoichman M, et al. Three-dimensional folding and functional organization principles of the Drosophila genome. Cell. 2012;148:458-72.

76. Gonzalez-Sandoval A, Gasser SM. On TADs and LADs: spatial control over gene expression. Trends Genet. 2016;32:485-95.

77. Barutcu AR, Lian JB, Stein JL, Stein GS, Imbalzano AN. The connection between BRG1, CTCF and topoisomerases at TAD boundaries. Nucleus. 2017;8:150-5

78. Alabert C, Bukowski-Wills JC, Lee SB, Kustatscher G, Nakamura K, de Lima AF, et al. Nascent chromatin capture proteomics determines chromatin dynamics during DNA replication and identifies unknown fork components. Nat Cell Biol. 2014;16:281-93.

79. Alabert C, Groth A. Chromatin replication and epigenome maintenance. Nat Rev Mol Cell Biol. 2012;13:153-67.

80. Liu Q, Gong Z. The coupling of epigenome replication with DNA replication. Curr Opin Plant Biol. 2011:14:187-94.

81. Gutierrez C, Desvoyes B, Vergara Z, Otero S, Sequeira-Mendes J. Links of genome replication, transcriptional silencing and chromatin dynamics. Curr Opin Plant Biol. 2016;34:92-9.
82. Gutierrez C, Sequeira-Mendes J, Araguez I. DNA replication of plant genomes. In: Tester M, Jorgensen RA, editors. Nucleus \& Genome. Dordrecht: Springer; 2013. doi:10.1007/978-1-4939-0263-7_1-1.

83. Barrero JM, Gonzalez-Bayon R, del Pozo JC, Ponce MR, Micol JL. INCURVATA2 encodes the catalytic subunit of DNA polymerase alpha and interacts with genes involved in chromatin-mediated cellular memory in Arabidopsis thaliana. Plant Cell. 2007;19:2822-38.

84. Hyun Y, Yun H, Park K, Ohr H, Lee O, Kim DH, et al. The catalytic subunit of Arabidopsis DNA polymerase alpha ensures stable maintenance of histone modification. Development. 2013;140:156-66.

85. Gong Z, Morales-Ruiz T, Ariza RR, Roldan-Arjona T, David L, Zhu JK. ROS1, a repressor of transcriptional gene silencing in Arabidopsis, encodes a DNA glycosylase/lyase. Cell. 2002;111:803-14.

86. Morales-Ruiz T, Ortega-Galisteo AP, Ponferrada-Marin MI, Martinez-Macias MI, Ariza RR, Roldan-Arjona T. DEMETER and REPRESSOR OF SILENCING 1 encode 5-methylcytosine DNA glycosylases. Proc Natl Acad Sci U S A. 2006:103:6853-8

87. Liu J, Ren X, Yin H, Wang Y, Xia R, Wang Y, Gong Z. Mutation in the catalytic subunit of DNA polymerase alpha influences transcriptional gene silencing and homologous recombination in Arabidopsis. Plant J. 2010;61:36-45.

88. Iglesias FM, Bruera NA, Dergan-Dylon S, Marino-Buslje C, Lorenzi H, Mateos $J$, et al. The arabidopsis DNA polymerase delta has a role in the deposition of transcriptionally active epigenetic marks, development and flowering. PLoS Genet. 2015;11:e1004975.

89. Zhang J, Xie S, Cheng J, Lai J, Zhu JK, Gong Z. The second subunit of DNA polymerase delta is required for genomic stability and epigenetic regulation. Plant Physiol. 2016;171:1192-208.

90. Jenik PD, Jurkuta RE, Barton MK. Interactions between the cell cycle and embryonic patterning in Arabidopsis uncovered by a mutation in DNA polymerase epsilon. Plant Cell. 2005;17:3362-77.

91. Yin H, Zhang X, Liu J, Wang Y, He J, Yang T, et al. Epigenetic regulation, somatic homologous recombination, and abscisic acid signaling are influenced by DNA polymerase epsilon mutation in Arabidopsis. Plant Cell. 2009;21:386-402.

92. del Olmo I, Lopez-Gonzalez L, Martin-Trillo MM, Martinez-Zapater JM, Pineiro M, Jarillo JA. EARLY IN SHORT DAYS 7 (ESD7) encodes the catalytic subunit of DNA polymerase epsilon and is required for flowering repression through a mechanism involving epigenetic gene silencing. Plant J. 2010;61:623-36.

93. del Olmo I, Lopez JA, Vazquez J, Raynaud C, Pineiro M, Jarillo JA. Arabidopsis DNA polymerase recruits components of Polycomb repressor complex to mediate epigenetic gene silencing. Nucleic Acids Res. 2016;44:5597-614.

94. Pedroza-Garcia JA, Domenichini S, Mazubert C, Bourge M, White C, Hudik E, et al. Role of the polymerase sub-unit DPB2 in DNA replication, cell cycle regulation and DNA damage response in Arabidopsis. Nucleic Acids Res. 2016;44:7251-66.

95. Pedroza-Garcia JA, Mazubert C, Del Olmo I, Bourge M, Domenichini S, Bounon $\mathrm{R}$, et al. Function of the Plant DNA polymerase epsilon in replicative stress sensing, a genetic analysis. Plant Physiol. 2017:173:1735-49.

96. Raynaud C, Sozzani R, Glab N, Domenichini S, Perennes C, Cella R, et al. Two cell-cycle regulated SET-domain proteins interact with proliferating cell nuclear antigen (PCNA) in Arabidopsis. Plant J. 2006;47:395-407.

97. Jacob Y, Feng S, LeBlanc CA, Bernatavichute $\mathrm{V}$, Stroud H, Cokus S, et al. ATXR5 and ATXR6 are H3K27 monomethyltransferases required for chromatin structure and gene silencing. Nat Struct Mol Biol. 2009;16:763-8.

98. Stroud H, Otero S, Desvoyes B, Ramirez-Parra E, Jacobsen SE, Gutierrez C. Genome-wide analysis of histone $\mathrm{H} 3.1$ and $\mathrm{H} 3.3$ variants in Arabidopsis thaliana. Proc Natl Acad Sci U S A. 2012;109:5370-5.

99. Wollmann H, Holec S, Alden K, Clarke ND, Jacques PE, Berger F. Dynamic deposition of histone variant H3.3 accompanies developmental remodeling of the Arabidopsis transcriptome. PLoS Genet. 2012;8:e1002658.

100. Jacob Y, Bergamin E, Donoghue MT, Mongeon V, LeBlanc C, Voigt P, et al. Selective methylation of histone $\mathrm{H} 3$ variant $\mathrm{H} 3.1$ regulates heterochromatin replication. Science. 2014:343:1249-53.

101. Jacob Y, Stroud H, Leblanc C, Feng S, Zhuo L, Caro E, et al. Regulation of heterochromatic DNA replication by histone $\mathrm{H} 3$ lysine 27 methyltransferases. Nature. 2010;466:987-91.

102. Hale CJ, Potok ME, Lopez JA, Do T, Liu A, Gallego-Bartolome J, et al. Identification of multiple proteins coupliing transcriptional gene-silencing to genome stability in Arabidopsis thaliana. PLoS Genet. 2016;12:e1006092.

103. Stroud H, Hale CJ, Feng S, Caro E, Jacob Y, Michaels SD, Jacobsen SE. DNA methyltransferases are required to induce heterochromatic re-replication in Arabidopsis. PLoS Genet. 2012:8:e1002808. 
104. Lombrana R, Almeida R, Alvarez A, Gomez M. R-loops and initiation of DNA replication in human cells: a missing link? Front Genet. 2015;6:158.

105. Devbhandari S, Jiang J, Kumar C, Whitehouse I, Remus D. Chromatin constrains the initiation and elongation of DNA replication. Mol Cell. 2017;65:131-41.

106. Kurat CF, Yeeles JT, Patel H, Early A, Diffley JF. Chromatin controls DNA replication origin selection, lagging-strand synthesis, and replication fork rates. Mol Cell. 2017;65:117-30.

107. Dona M, Mittelsten SO. DNA damage repair in the context of plant chromatin. Plant Physiol. 2015;168:1206-18.

108. Hu Z, Cools T, De Veylder L. Mechanisms used by plants to cope with DNA damage. Annu Rev Plant Biol. 2016;67:439-62.

109. Spampinato CP. Protecting DNA from errors and damage: an overview of DNA repair mechanisms in plants compared to mammals. Cell Mol Life Sci. 2017;74:1693-709.

110. Drury GE, Dowle AA, Ashford DA, Waterworth WM, Thomas J, West CE. Dynamics of plant histone modifications in response to DNA damage. Biochem J. 2012;445:393-401.

111. Questa JI, Fina JP, Casati P. DDM1 and ROS1 have a role in UV-B inducedand oxidative DNA damage in A. thaliana. Front. Plant Sci. 2013;4:420.

112. Scharer OD. Nucleotide excision repair in eukaryotes. Cold Spring Harb Perspect Biol. 2013;5:a012609.

113. Schalk C, Drevensek S, Kramdi A, Kassam M, Ahmed I, Cognat V, et al. DNA DAMAGE BINDING PROTEIN 2 (DDB2) shapes the DNA methylation landscape. Plant Cell. 2016;28:2043-59.

114. Yao Y, Bilichak A, Golubov A, Kovalchuk I. ddm 1 plants are sensitive to methyl methane sulfonate and $\mathrm{NaCl}$ stresses and are deficient in DNA repair. Plant Cell Rep. 2012;31:1549-61.

115. Mondal S, Go YS, Lee SS, Chung BY, Kim JH. Characterization of histone modifications associated with DNA damage repair genes upon exposure to gamma rays in Arabidopsis seedlings. J Radiat Res. 2016;57:646-54.

116. Zhou W, Gao J, Ma J, Cao L, Zhang C, Zhu Y, et al. Distinct roles of the histone chaperones NAP1 and NRP and the chromatin-remodeling factor INO80 in somatic homologous recombination in Arabidopsis thaliana. Plant J. 2016:88:397-410.

117. Martinez-Fabregas J, Diaz-Moreno I, Gonzalez-Arzola K, Janocha S, Navarro JA, Hervas M, et al. New Arabidopsis thaliana cytochrome c partners: a look into the elusive role of cytochrome $\mathrm{c}$ in programmed cell death in plants. Mol Cell Proteomics. 2013;12:3666-76.

118. Gonzalez-Arzola K, Diaz-Quintana A, Rivero-Rodriguez F, Velazquez-Campoy A, De la Rosa MA, Diaz-Moreno I. Histone chaperone activity of Arabidopsis thaliana NRP1 is blocked by cytochrome c. Nucleic Acids Res. 2017:45:2150-65.

119. Martinez-Fabregas J, Diaz-Moreno I, Gonzalez-Arzola K, Janocha S, Navarro JA, Hervas M, et al. Structural and functional analysis of novel human cytochrome C targets in apoptosis. Mol Cell Proteomics. 2014;13:1439-56.

120. Gonzalez-Arzola K, Diaz-Moreno I, Cano-Gonzalez A, Diaz-Quintana A, Velazquez-Campoy A, Moreno-Beltran B, et al. Structural basis for inhibition of the histone chaperone activity of SET/TAF-lbeta by cytochrome c. Proc Natl Acad Sci U S A. 2015;112:9908-13.

121. Rosa M, Von Harder M, Cigliano RA, Schlogelhofer P, Mittelsten SO. The Arabidopsis SWR1 chromatin-remodeling complex is important for DNA repair, somatic recombination, and meiosis. Plant Cell. 2013;25:1990-2001.

122. Torres-Rosell J, Sunjevaric I, De Piccoli G, Sacher M, Eckert-Boulet N, Reid R, et al. The Smc5-Smc6 complex and SUMO modification of Rad52 regulates recombinational repair at the ribosomal gene locus. Nat Cell Biol. 2007:9:923-31.

123. Feng W, Hale CJ, Over RS, Cokus SJ, Jacobsen SE, Michaels SD. Large-scale heterochromatin remodeling linked to overreplication-associated DNA damage. Proc Natl Acad Sci U S A. 2017;114:406-11.

124. Dabin J, Fortuny A, Polo SE. Epigenome maintenance in response to DNA damage. Mol Cell. 2016;62:712-27.

125. Yelagandula R, Stroud H, Holec S, Zhou K, Feng S, Zhong X, et al. The histone variant H2A.W defines heterochromatin and promotes chromatin condensation in Arabidopsis. Cell. 2014;158:98-109.

126. Lorkovic ZJ, Park C, Goiser M, Jiang D, Kurzbauer MT, Schlogelhofer P, Berger F. Compartmentalization of DNA damage response between heterochromatin and euchromatin is mediated by distinct $\mathrm{H} 2 \mathrm{~A}$ histone variants. Curr Biol. 2017;27:1192-9.

127. Yelina NE, Lambing C, Hardcastle TJ, Zhao X, Santos B, Henderson IR. DNA methylation epigenetically silences crossover hot spots and controls chromosomal domains of meiotic recombination in Arabidopsis. Genes Dev. 2015:29:2183-202.
128. Zhang J, Xie S, Zhu JK, Gong Z. Requirement for flap endonuclease 1 (FEN1) to maintain genomic stability and transcriptional gene silencing in Arabidopsis. Plant J. 2016;87:629-40.

129. Bourbousse C, Mestiri I, Zabulon G, Bourge M, Formiggini F, Koini MA, et al. Light signaling controls nuclear architecture reorganization during seedling establishment. Proc Natl Acad Sci U S A. 2015;112:E2836-44.

130. Sanchez DH, Paszkowski J. Heat-induced release of epigenetic silencing reveals the concealed role of an imprinted plant gene. PLoS Genet. 2014;10:e1004806.

131. Mozgova I, Köhler C, Hennig L. Keeping the gate closed: functions of the polycomb repressive complex PRC2 in development. Plant J. 2015;83:121-32.

132. Brady SM, Orlando DA, Lee JY, Wang JY, Koch J, Dinneny JR, et al. A highresolution root spatiotemporal map reveals dominant expression patterns. Science. 2007;318:801-6.

133. Berger F, Twell D. Germline specification and function in plants. Annu Rev Plant Biol. 2011:62:461-84.

134. Bustamante M, Matus J, Riechmann JL. Genome-wide analyses for dissecting gene regulatory networks in the shoot apical meristem. J Exp Bot. 2016;67:1639-48. 